\title{
Route Capacity Estimation Based Admission Control and QoS Routing for Mesh Networks
}

\author{
Chi Harold Liu, Athanasios Gkelias, and Kin K. Leung \\ Department of Electrical and Electronic Engineering \\ Imperial College \\ Exhibition Road, London, SW7 2BT, United Kingdom \\ \{chiliu, a.gkelias, kin.leung\}@imperial.ac.uk
}

\begin{abstract}
Wireless Mesh Networks (WMNs) is a promising key technology for next generation wireless backhauling that is expected to support various types of applications with different quality-of-service (QoS) demands. Advanced antenna techniques, novel scheduling algorithms and routing schemes have attracted increased research interests aiming to optimize the performance of WMNs and satisfy their vast and diverse traffic requirements. However, in such networks, uncontrolled admission of arbitrary large number of data flows, together with the distributed and dynamic nature of WMNs, can highly increase the wireless channel interference with catastrophic subsequence for the whole network. To overcome this difficulty, we provide a mathematical model to analyze and estimate the real-time "route capacity" associated with time-varying QoS requirements. This allows us to take admission control decisions for new data flows in a way that we can increase the resource utilization while maintaining all good QoS levels for users with different grade-of-service (GoS). Numerical and extensive simulations results show that the proposed scheme achieves higher network goodput while it significantly reduces the outage and session blocking probability if compared to other techniques.
\end{abstract}

\section{INTRODUCTION}

The recent advance of wireless networking technologies has prompted a flourish of a new kind of multi-hop wireless network architecture, i.e., wireless mesh networks (WMNs, [1]). Their popularity comes from the fact that they are selforganized, self-configurable and easily adaptable to different traffic requirements and network changes. WMNs are composed of static wireless nodes that have ample energy supply. Each node operates not only as a conventional access point/gateway to the internet but also as a wireless router able to relay packets from other nodes without direct access to their destinations. The destination can be an internet gateway or a mobile user served by another AP in the same mesh network. Moreover, some nodes may only have the backhauling functionality, meaning that they do not serve any mobile user directly but their purpose is to forward other APs' packets.

This research was partly financially supported by the EU IST FP6 MEMBRANE project (contract number: 027310). This research was also partly sponsored by US Army Research laboratory and the UK Ministry of Defence and was accomplished under Agreement Number W911NF-06-30001. The views and conclusions contained in this document are those of the authors and should not be interpreted as representing the official policies, either expressed or implied, of the US Army Research Laboratory, the U.S. Government, the UK Ministry of Defense, or the UK Government. The US and UK Governments are authorized to reproduce and distribute reprints for Government purposes notwithstanding any copyright notation hereon.
WMNs are expected to provide backhaul support to a wide range of applications including multimedia data services (e.g., voice-over-IP, interactive video, remote access and gaming, etc) with multiple, usually strict, QoS requirements. On the other hand, telecommunication service provider and operators expect to be able to provide different grade-of-services (such as, premium and regular services) that are adaptable to various billing systems in order to improve the user experience while at the same time maximize their revenue (e.g., higher GoS can be obtained for premium users at a higher cost). This imposes another dimension of difficulty for resource allocation, if fairness and priorities should be maintained simultaneously among all users.

While several solutions for QoS and GoS support have been proposed for centralized networks, the inherent differences between centralized and distributed communications necessitate the design of new solutions for WMNs. The main difficulties arise from the highly dynamic and multi-hop nature of mesh networks. In such networks, proportional fair scheduling (PFS) has been proven to greatly improve the overall network throughput by exploiting the multi-user diversity gain. However, due to its opportunistic nature, it is unable to provide hard resource reservations required to guarantee QoS to the underlying applications. For instance, increased resource demand from higher layers will impose increased pressure to the scheduler forcing it to take suboptimum decisions. Scheduling suboptimal links for transmission, increased queuing delays resulting to buffer overflows and failure for delay critical applications and increased interferences to/from adjacent links are some of the consequences of route overloading.

Therefore, it is highly important the design of an admission control scheme that exploits the information gathered by different layers to avoid the aforementioned drawbacks and minimize the QoS outage and negative impact of new flows on the existing ones. However, in a multi-hop wireless environment, the dynamic traffic load, route selection, packet scheduling, channel fluctuation etc. make the "route capacity" unpredictable and time varying. Given these design requirements, unfortunately, not much research work so far has successfully addressed the problem.

In this paper, we propose a joint connection admission control (CAC) and QoS/GoS routing framework for WMNs. As a first step, we provide a mathematical model to estimate in 
real-time the available resources (hereafter referred to as "route capacity") of any given route and the impact of any given data flow with certain QoS requirements on this route. This allows designing a CAC algorithm at the source level, that exploits the estimated route capacity information and takes decisions based on the QoS and GoS application requirements.

\section{RELATED WORK}

Recently, much work has been done on admission control algorithms in ad hoc wireless networks [2]; but not much work has been done to provide both QoS and GoS provisions for traffics with multiple patterns in WMNs. [3] proposed an admission control algorithm for connections with rate and delay requirements in wireless backhaul networks. However, it assumes no channel fading and co-channel interferences among wireless links, and uses a tree-structure [4] MAC scheduling. In [5], a joint centralized scheduling and time slot allocation based admission control algorithm is proposed for WiMAX networks, which allows to admit a flow if extra unused slots are sufficient to satisfy bandwidth requirement. The integrated framework of routing and admission control for IEEE 802.16 distributed mesh networks was studied in [6]. It estimates available bandwidth in a token bucket to perform admission control with minimum time slot requirement for each connection, and it uses shortest-widest efficient bandwidth metric for route discovery. This work cannot provide real-time QoS provisions and no actual interface between routing and admission control. Similarly, in ad-hoc network settings, [7] proposed an AODV routing protocol based admission control, whereas it blocks the over-loaded flow requests during the routing discovery procedure. [8] is much related to our work that makes admission decision by estimating the achievable capacity between any pair of ingress and egress nodes with only packet loss constraint, assuming traffics arrive according to Gaussian distribution. However, in a multi-hop wireless network, multiple joint/disjoint routes exist between any pair of source and destination, thus it is more appropriate and accurate to make admission decision for each route. Moreover, Gaussian traffic arrival assumption may not always hold, thus achievable capacity region could be inaccurate. Works [9], [10] study extensively on the integrated QoS routing protocol and the actual interface between the scheduling [11] and routing schemes, to provide optimum routes that guarantee multiple QoS constraints.

In this paper, we propose a route capacity estimated based connection admission control algorithm to provide multi-QoS constrains for users with different GoS. Our contributions are threefold:

(1) We treat each candidate route as a "black box", while using a generic mathematical function to represent the core resource manager. Considering traffic statistics as multiple input variables, QoS performance index as an output, we propose an analytical model to estimate the time-varying "route capacity" with accuracy in real-time.

(2) By estimating the route capacity, a distributed connection admission control scheme is proposed. It selects the route with minimum achievable QoS performance index made to existing flows if accepted on that route, or it minimizes the negative effects of new flows on the existing ones, i.e., the outage probability.

(3) We explicitly consider users with different GoS requirements from network operation perspective, while trying to allocate adequate resources for all users.

The rest of the paper is organized as follows. In Section III, the network model and QoS routing algorithm are briefly introduced. Section IV introduces the analytical model for estimating the "route capacity". Later, a thorough description of the proposed admission control scheme is proposed in Section V. Finally, numerical results and detailed analysis are given in Section VI and conclusions are drawn in Section VII.

\section{Network Model And QoS Routing Algorithm}

Consider a WMN comprises a set of $n_{r}$ mesh routers, as $V_{R}=\left\{v_{r} \mid r=1,2, \ldots, n_{r}\right\}$. The network runs under a timedivision multiple access (TDMA) slotted framework, where a time frame consists of $f_{d}$ fixed-size time slots for data, and $f_{c}$ fixed-size time slots for control messages. During the period of one time frame, we assume block fading channel that remains relatively constant. Scheduling decisions are taken by all nodes in the network in a distributed way at the beginning of each time frame at the control phase, and stay unchanged until the next frame. The PHY layer employs the adaptive modulation and coding techniques, where there are a finite number $V$ of transmission modes, each of which corresponds to a unique modulation and coding scheme and one particular interval of the received signal to interference plus noise ratio (SINR). The transmission rate at each mode is proportional to its spectral efficiency, i.e., transmission mode $v$ can transmit maximum $c_{v}$ packets in one time slot, where $v=1,2, \ldots, V$. Furthermore, in order to reduce the interference to adjacent concurrent transmissions, increase the frequency reuse and channel capacity, the nodes are equipped with directional antennas.

QoS flow $q$ is generated with a set of constraints, ETE packet delay, throughput and PER. Our previous results in [9], [10] defined a new utility function based on the "dissatisfaction ratio" $\mathbf{R}$ that is experienced by each QoS metric, $\mathbf{R}_{k}^{D}$ for ETE packet delay, $\mathbf{R}_{k}^{T}$ for throughput, and $\mathbf{R}_{k}^{E}$ for PER. Hence, a source-to-gateway route $k$ will be feasible if and only if all defined ratios are less than 1 , i.e., $\left(\mathbf{R}_{k}^{D}, \mathbf{R}_{k}^{T}, \mathbf{R}_{k}^{E}\right) \leq 1$. Later, associated QoS performance index $\mathbf{I}_{s g}^{k}$ can be formulated as,

$$
\mathbf{I}_{s g}^{k}=\max \left[\mathbf{R}_{k}^{D}, \mathbf{R}_{k}^{T}, \mathbf{R}_{k}^{E}\right] \leq 1
$$

and the optimum route selection decision is given by $\min _{\forall k \in \Omega_{s g}}\left[\mathbf{I}_{s g}^{k}\right]$, where $\Omega_{s g}$ is the route set from a source $s$ to a destination $g$.

Furthermore, our routing algorithm requires every node $s$ in the network keeps track of connection level statistics in each route $k$, including the number of ongoing connections $N_{s g}^{k}(t)$, total guaranteed throughout $T_{s g}^{k}(t)$, and the observable QoS performance index $\mathbf{I}_{s g}^{k}(t)$ fed back by corresponding 


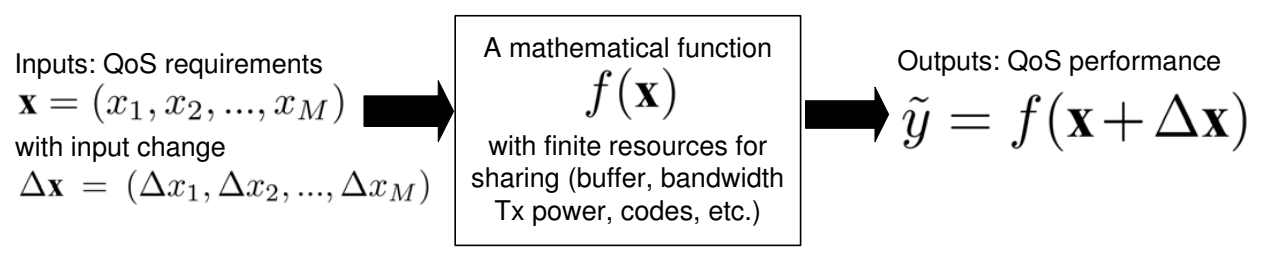

Fig. 1. A mathematical representation of each candidate route between any source and gateway pair, where core resource allocation manager (buffer, bandwidth, Transmission (Tx) power, codes, etc.) is treated as a black box. Source node's $M$ dimension input variables represent traffic statistics such as QoS requirement, burstiness, session duration etc., and observable QoS performance index is a single gateway output.

gateway $g$. These information will be collected and updated periodically similar to routing operation time scales.

\section{Route CAPACity Estimation}

Opportunistic scheduling can take advantage of the multiuser diversity gain and the dynamic nature of the wireless channel, however, it comes with a certain drawback, i.e., while it maximizes the overall network throughput it cannot perform hard resource reservation that is required to provide strict QoS for each session. As a result, an increased outage probability of the ongoing QoS sessions is expected.

Therefore, a scheme is required to provide connection admission control to new flows by predicting their impact on the QoS of the flows already running in the network. Consider a node $s$, serves as the source expected to route data to gateway $g$. The basic question to ask is whether any route could provide enough available resources to support the required QoS. These resources can be any combination of buffer, time, transmission power, codes etc, but all those could be interpreted as a form of end-to-end wireless bandwidth, or so-called "route capacity". Unfortunately, this route capacity has been well-known very difficult to estimate in WMNs, because sessions have timevarying, stochastic resource demands from application layer, a degree of freedom for route selection in the network layer, packet switching, power control in MAC layer, and large variability of data rates for users in different wireless channel conditions. As a result, even of same type of application requests, they would consume different amount of resources at different time, let alone in a case where voice, video, data services will arrive in an unpredicted manner.

We model the whole network as a set of source/gateway communication pairs, where each pair is comprised of multiple candidate routes. For each route, protocol layer 1, 2, and 3 control the resource allocation and management for all connections running on it to maintain certain $\mathrm{QoS}$ requirements, but these hop by hop operational characteristics along the entire route of communication are not apparent to end node upper layers. Therefore, we pick individual candidate route as the concept of "wireless system", and treat this system as a black box as shown in Fig.1, where finite resources are shared by multiple connections. As said, these resources include buffer, bandwidth, transmission power, codes, etc. The system inputs are arrival connection requirements and associated traffic characteristics such as QoS requirements including throughput, delay and PER. By monitoring the resource occupancy, we want to identify the potentially limiting resources and estimate the route capacity to decide how many connections or how much flow of various QoS requirements can be supported simultaneously at a particular time $t$.

Without loss of generality, we use a mathematical function $f$ to represent the core resource manager to map inputs to an output. It is worth noting that $f$ is usually a complicated function, which generally cannot be expressed in close-form equations or formulas. Let vector $\mathbf{x}=\left(x_{1}, x_{2}, \ldots, x_{M}\right)$ denotes the $M$ input variables, and $y=f(\mathbf{x})$ denotes the output degree of resource occupancy. These consist of $M+1$ dimensional space as shown in Fig. 2(a), where $M$ input dimensions may not be orthogonal but represent the required traffic statistics. To overcome the difficulties of close-form representation of $f$, we use Taylor expansion of $f$ to approximate this resource manager. Because the connection admission control scheme wants to answer the basic question of acceptance/rejection of new incoming flows, we characterize the potential admission as an input change $\Delta \mathbf{x}=\left(\Delta x_{1}, \Delta x_{2}, \ldots, \Delta x_{M}\right)$ into the resource manager, which will result in an output change of $\tilde{y}=f(\mathbf{x}+\Delta \mathbf{x})$. Then, if we only take the first and second order partial derivatives of $f$, we have the following approximation,

$$
\begin{aligned}
& \tilde{y}(\mathbf{x}+\Delta \mathbf{x}) \approx f(\mathbf{x})+\sum_{i=1}^{M} f_{x_{i}}^{\prime} \Delta x_{i} \\
& +\frac{1}{2 !}\left\{\sum_{i=1}^{M} f_{x_{i}}^{\prime \prime} \Delta x_{i}^{2}+\sum_{i=1}^{M} \sum_{j \neq i} f_{x_{j} x_{i}}^{\prime} \Delta x_{i} \Delta x_{j}\right\}
\end{aligned}
$$

where we denote $f_{x_{i}}^{\prime}=\frac{\partial f}{\partial x_{i}}, f_{x_{i}}^{\prime \prime}=\frac{\partial f^{2}}{\partial x_{i}^{2}}$. Before we proceed, we need to prove the validity of neglecting all higher order Taylor series. This is because the route capacity is usually much larger than resource demands of one single connection, and thus the admission or completion of one connection do not drastically change the overall session performances, and any change in the overall session performances due to admission or completion of one connection is adequately reflected in the measured output before the next admission event that could be maintained by monitoring enough connections before making the decisions. For one dimension of input variable and corresponding output, through real-time statistics collection and monitoring, we could have Fig. 2(b)-like relations.

For simplicity reasons, consider a specific route $k$ between $s-g$ pair, further use $N_{s g}^{k}(t)$ and $N_{t}, T_{s g}^{k}(t)$ and $T_{t}, \mathbf{I}_{s g}^{k}(t)$ and $\mathbf{I}_{t}$ interchangeably. In the following discussions, we use the number of existing connections $N_{t}$ and total guaranteed throughput $T_{t}$ along the route as input variables (i.e., $M=2$ ), 
use observable QoS performance index $\mathbf{I}_{t}$ associated as the output, i.e.,

$$
\mathbf{I}_{t}=f\left(N_{t}, T_{t}\right)
$$

If the current route operates at $\left(N_{t_{0}}, T_{t_{0}}\right)$ at time $t_{0}$ when the new connection arrives for admission decision, i.e., $N_{t_{0}}$ number of existing connections with total aggregated throughput $T_{t_{0}}$ already running in the network, we are interested to know the maximum aggregated throughput $C_{t_{0}^{+}}$the route can support at time $t=t_{0}^{+}$that corresponds to the maximum tolerable QoS performance index 1. Or, given the input change $\Delta N=1, \Delta T=C_{t_{0}^{+}}-T_{t_{0}}$, for the output change,

$$
\tilde{\mathbf{I}}_{t_{0}^{+}}\left(N_{t_{0}}+1, C_{t_{0}^{+}}\right)=1
$$

should have to hold. Therefore, we rewrite (2) as,

$$
\begin{gathered}
\tilde{\mathbf{I}}_{t_{0}^{+}}\left(N_{t_{0}}+1, C_{t_{0}^{+}}\right)=\mathbf{I}_{t_{0}}+\left.\Delta N f_{N_{t}}^{\prime}\right|_{t=t_{0}}+\left.\Delta T f_{T_{t}}^{\prime}\right|_{t=t_{0}} \\
+\left.\frac{\Delta N^{2}}{2} f_{N_{t}}^{\prime \prime}\right|_{t=t_{0}}+\left.\frac{\Delta T^{2}}{2} f_{T_{t}}^{\prime \prime}\right|_{t=t_{0}}+\left.\Delta N \Delta T f_{N_{t} T_{t}}^{\prime \prime}\right|_{t=t_{0}}
\end{gathered}
$$

or,

$$
\begin{array}{r}
1=\mathbf{I}_{t_{0}}+\left.f_{N_{t}}^{\prime}\right|_{t=t_{0}}+\left.\left(C_{t_{0}^{+}}-T_{t_{0}}\right) f_{T_{t}}^{\prime}\right|_{t=t_{0}}+\left.\frac{1}{2} f_{N_{t}}^{\prime \prime}\right|_{t=t_{0}} \\
+\left.\frac{\left(C_{t_{0}^{+}}-T_{t_{0}}\right)^{2}}{2} f_{T_{t}}^{\prime \prime}\right|_{t=t_{0}}+\left.\left(C_{t_{0}^{+}}-T_{t_{0}}\right) f_{N_{t} T_{t}}^{\prime \prime}\right|_{t=t_{0}}
\end{array}
$$

In order to get the second-order partial derivative $f_{N_{t} T_{t}}^{\prime \prime}$, we notice that the chosen input variables $T_{t}$ and $N_{t}$ are correlated by $T_{t}=\sum_{q=1}^{N_{t}} T_{q}^{r}$, where $T_{q}^{r}$ is the throughput requirement for each ongoing session $q$. Then, by using the limitation expression of first-order partial derivative, we have,

$$
\begin{aligned}
& \left.\frac{\partial T_{t}}{\partial N_{t}}\right|_{t=t_{0}}=\lim _{\Delta N \rightarrow 1} \frac{\sum_{q=1}^{N_{t_{0}}+\Delta N} T_{q}^{r}-\sum_{q=1}^{N_{t_{0}}} T_{q}^{r}}{\Delta N} \\
& =\lim _{\Delta N \rightarrow 1} \frac{\sum_{q=N_{t_{0}}+1}^{N_{t_{0}}+\Delta N} T_{q}^{r}}{\Delta N}=T_{t_{0}^{+}}^{r}=C_{t_{0}^{+}}-T_{t_{0}}
\end{aligned}
$$

where $T_{t_{0}^{+}}^{r}$ denotes the incoming input connection throughput requirement at time $t=t_{0}^{+}$. Therefore,

$$
\left.f_{N_{t} T_{t}}^{\prime \prime}\right|_{t=t_{0}}=\left.f_{T_{t}}^{\prime \prime} \frac{\partial T_{t}}{\partial N_{t}}\right|_{t=t_{0}}=\left.\left(C_{t_{0}^{+}}-T_{t_{0}}\right) f_{T_{t}}^{\prime \prime}\right|_{t=t_{0}}
$$

Hence we rewrite (6) as,

$$
\begin{aligned}
& \frac{1}{2} f_{N_{t}}^{\prime \prime}+\frac{3\left(C_{t_{0}^{+}}-T_{t_{0}}\right)^{2}}{2} f_{T_{t}}^{\prime \prime}+f_{N_{t}}^{\prime}+\left(C_{t_{0}^{+}}-T_{t_{0}}\right) f_{T_{t}}^{\prime} \\
& +\mathbf{I}_{t_{0}}-1=0
\end{aligned}
$$

where all partial derivatives are taken at point $\left(N_{t_{0}}, T_{t_{0}}\right)$. By solving quadratic function (9), we show that the route capacity is approximated by (10).

$$
C_{t_{0}^{+}}=\frac{\sqrt{f_{T_{t}}^{\prime 2}-6 f_{T_{t}}^{\prime \prime}\left(f_{N_{t}}^{\prime}+\frac{f_{N_{t}}^{\prime \prime}}{2}+\mathbf{I}_{t_{0}}-1\right)}-f_{T_{t}}^{\prime}}{3 f_{T_{t}}^{\prime \prime}}+T_{t_{0}}
$$

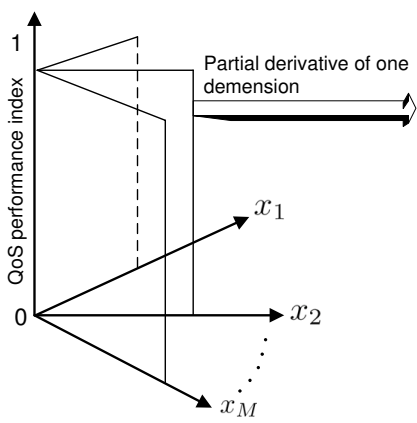

(a)

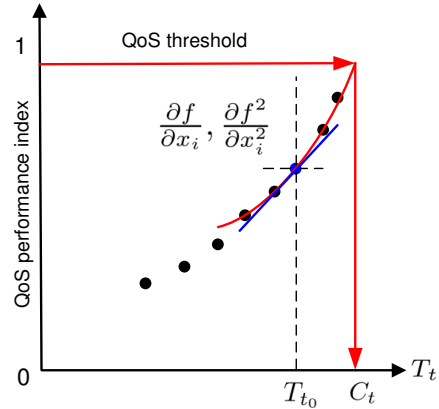

(b)
Fig. 2. (a) $M+1$ dimension space representation of wireless system, (b) An example of how to obtain partial derivatives and route capacity estimation based on measurements.

If further assuming that $f$ is smooth enough around point $\left(N_{t_{0}}, T_{t_{0}}\right)$, i.e., $\exists \varepsilon \approx 0$, and $\delta>0$, so that,

$$
\begin{aligned}
& \operatorname{Pr}\left(\left|f_{N_{t}}^{\prime \prime}\right| \geq \delta\right)<\varepsilon \\
& \operatorname{Pr}\left(\left|f_{N_{t} T_{t}}^{\prime \prime}\right| \geq \delta\right)<\varepsilon \\
& \operatorname{Pr}\left(\left|f_{T_{t}}^{\prime \prime}\right| \geq \delta\right)<\varepsilon
\end{aligned}
$$

which means the second-order derivatives are neglectable, we simplify (10) as:

$$
C_{t_{0}^{+}}=\frac{1-\mathbf{I}_{t_{0}}-f_{N_{t}}^{\prime}}{f_{T_{t}}^{\prime}}+T_{t_{0}} .
$$

To sum up, given the generic mathematical function as the resource manager, consider the number of connections and total guaranteed throughput of current running connections as the input variables, and observable QoS performance index as the output, the time varying "route capacity" could be obtained by a close-form approximation of only first and second order partial derivatives $\left.f_{T_{t}}^{\prime}\right|_{t=t_{0}},\left.f_{T_{t}}^{\prime \prime}\right|_{t=t_{0}},\left.f_{N_{t}}^{\prime}\right|_{t=t_{0}},\left.f_{N_{t}}^{\prime \prime}\right|_{t=t_{0}}$. On the other hand, if the function $f$ is smooth enough at the operating point $\left(N_{t_{0}}, T_{t_{0}}\right)$ so that the second-order statistics are neglectable, this capacity could further be simplified by using only first-order derivatives $\left.f_{T_{t}}^{\prime}\right|_{t=t_{0}},\left.f_{N_{t}}^{\prime}\right|_{t=t_{0}}$.

This route capacity estimation scheme is fully distributed for every wireless mesh router in the network, and no global information is required. Moreover, it is easily expanded to other input variables besides $N_{t}$ and $T_{t}$ to give more accurate representation of the traffics, such as more complicated traffic characteristics like delay bound, jitter, packet length and burstiness, etc.

\section{Connection Admission Control Algorithm}

Our proposed connection admission control scheme is initialized when new session $q$ arrives at source node $s$ with multiple QoS constraints at time $t_{0}$, intended to communicative with gateway $g$. The functionality of the proposed algorithm is summarized and described in the following three steps.

Step-1: The source node $s$ implements route capacity estimation for every existing route $k$ between source-gateway pair $s-g$. Simply by measuring the first and second order statistics $\left.f_{T_{t}}^{\prime}\right|_{t=t_{0}},\left.f_{T_{t}}^{\prime \prime}\right|_{t=t_{0}},\left.f_{N_{t}}^{\prime}\right|_{t=t_{0}},\left.f_{N_{t}}^{\prime \prime}\right|_{t=t_{0}}$ at current point $t=t_{0}$, the capacity is approximated by (10). The estimated 
capacity $C_{t_{0}^{+}}^{k}$ indicates the maximum traffic load the route $k$ could support given the constraints that none of the existing ongoing flows will not be jeopardized for QoS.

Step-2: This step is to verify if enough resources on each route $k$ are available for the new incoming session. To differentiate different GoS, we notice that this session belongs to one of the two different service classes, regular or premium service. We introduce capacity usage indicators to provide capacity usage priorities for different GoS. They are: $\alpha_{r}^{H}, \alpha_{r}^{L}, \alpha_{p}^{H}, \alpha_{p}^{L}$ that denote the higher and lower bounds of overall capacity each service class can use respectively. Especially lower bound $\alpha_{p}^{L}$ reserves certain amount of overall route capacity for higher-priority users to guarantee certain QoS, which cannot be shared with regular services even though they starve. The typical values of these bounds are $\alpha_{r}^{H}=0.8, \alpha_{r}^{L}=0, \alpha_{p}^{H}=0.5, \alpha_{p}^{L}=0.2$.

Therefore, if the incoming session belongs to premium user group, available capacity for the new session is calculated by $\alpha_{p}^{H} C_{t_{0}^{+}}^{k}-T_{t}^{k, p}$, where $T_{t}^{k, p}$ denotes the total guaranteed throughput for ongoing premium sessions on route $k$. Similar judgement is easy to obtain for regular users. The admission decision variable $\Upsilon$ is chosen correspondingly as,

$$
\Upsilon^{k}= \begin{cases}1, & \text { if } T_{q}^{r} \leq \alpha_{p}^{H} C_{t_{0}^{+}}^{k}-T_{t}^{k, p}, \text { premium serv. } \\ 1, & \text { if } T_{q}^{r} \leq \alpha_{r}^{H} C_{t_{0}^{+}}^{k}-T_{t}^{k, r}, \text { regular serv. } \\ 0, & \text { otherwise }\end{cases}
$$

If admission decision variable $\Upsilon^{k}=0, \forall k$, it fails finding any route providing required QoS. Then, the source node initiates route discovery to search for new candidate routes that can provide QoS to the session. Meanwhile, Step-3 is ignored. If admission decision variable $\Upsilon^{k}=1, \exists k$, the load balancing function is called in Step-3.

Step-3: Although we may find multiple routes to be chosen as the candidate for the new flow, these routes may highly likely overlap with each other. Therefore, improper route selection may create bottlenecks in the network, thus deteriorate the overall performances.

Remind that by monitoring the first and second order partial derivatives of the number of existing connections and total guaranteed throughput $\left.f_{N_{t}}^{\prime}\right|_{t=t_{0}},\left.f_{T_{t}}^{\prime}\right|_{t=t_{0}},\left.f_{N_{t}}^{\prime \prime}\right|_{t=t_{0}},\left.f_{T_{t}}^{\prime \prime}\right|_{t=t_{0}}$ at current point $t=t_{0}$, we could estimate the QoS performance index if admitting the flow $q$ on the route $k$, as:

$$
\widehat{\mathbf{I}}_{t}^{k}=f\left(N_{t_{0}}^{k}+1, T_{t_{0}}^{k}+T_{q}^{r}\right)
$$

Let $\mathrm{I}\left(k^{\prime}\right)$ denote the set of QoS performance indexes if route $k^{\prime}$ is chosen to be the candidate route, as $\mathrm{I}\left(k^{\prime}\right)=$ $\left\{\mathbf{I}_{t}^{1}, \mathbf{I}_{t}^{2}, \ldots, \widehat{\mathbf{I}}_{t}^{k^{\prime}}, \ldots\right\}, \forall k^{\prime} \in \Omega_{s g}$. Then the load balancing algorithm will choose the route achieving the minimum variance among all $\mathbf{I}\left(k^{\prime}\right), \forall k^{\prime}$, which could be formally expressed as,

$$
\min _{\forall k^{\prime} \in \Omega_{s g}} \operatorname{var} \mathrm{I}\left(k^{\prime}\right)
$$

where we suppose route $k^{*}$ is finally chosen to be the candidate route. This route could minimize the overall impacts on both existing traffics of route $k^{*}$ and other joint routes between the associated source-gateway pair.

\section{NumericAl Results}

We developed a slotted, event-driven cross-layer OPNET [12] simulator to assess the proposed unified framework of multi-constrained QoS routing and connection admission control scheme with GoS guarantees. A number of wireless mesh routers are randomly and independently deployed, some of which have gateway functionalities. QoS Sessions are generated with three QoS constraints, i.e., ETE packet delay, throughput and PER are attached with each flow. Furthermore, sessions are randomly chosen to be either premium or regular service class. In network layer, the integrated QoS routing protocol [9], [10] is used to provide sub-optimal candidate routes with multiple QoS constraints. MAC layer uses distributed opportunities scheduler developed in [11]. In PHY Layer, the Rayleigh fading channel model [13] is used for the wireless channel representation while the required PER is derived based on SINR curves for the used adaptive modulation and coding scheme. Finally, the WMRs are equipped with directional antennas.

The proposed algorithm, referred as "Dist+IQoSR+RCCAC", is compared with our previous work presented in [9], [10] which does not include different GoS and prediction scheme for admission control "Dist+IQoSR". We also compare our scheme with conventional layer 2 and 3 techniques, the round robin scheduler (RR, [14]) and AODV routing protocol [15], and also the recent research output "SCAC" in [8] as benchmarks. The overall performances are investigated in terms of gateway goodput (Fig. 3a), average QoS outage probability of existing sessions (Fig. 3b), and session blocking probabilities for premium and regular users (Fig. 3c).

Fig. 3a shows that "Dist+IQoSR+RC-CAC" outperforms all other schemes in terms of overall gateway goodput. An important observation is that the proposed framework can successfully achieve high goodput even for small traffic interarrival rate (heavy load conditions), i.e., 1.4 times more than "Dist+IQoSR+SCAC", 2.2 times more than "Dist+IQoSR", and 3.2 times more than "RR+AODV". This is primarily because that the connection admission control scheme can admit or reject new sessions to maximize the end-to-end resource utilization in the network range by predicting the route capacity. By monitoring the resource occupancies along each route, it accurately identifies the potentially limiting resources and captures the impact of new arrival flows on the existing ongoing sessions. This will result in lower QoS outage probability (Fig. 3b) and session blocking probability (Fig. 3c), especially when the network operates at near-capacity region. Meanwhile, the GoS management allows certain bandwidth resources preserved for higher-level users. On the other hand, "SCAC" achieve high goodput when the traffic load is high due to its Gaussian traffic arrival assumptions, but when the traffic load is relatively low, it could not accurately estimate the achievable capacity region, thus make wrong decisions on flow admission which turns into less goodput, higher QoS outage.

Fig. 3b illustrates the probability of QoS outage of all completed sessions as a function of the traffic load. This is defined 


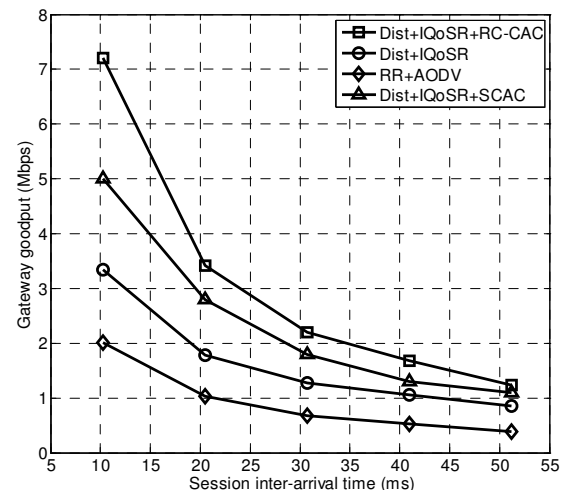

(a)

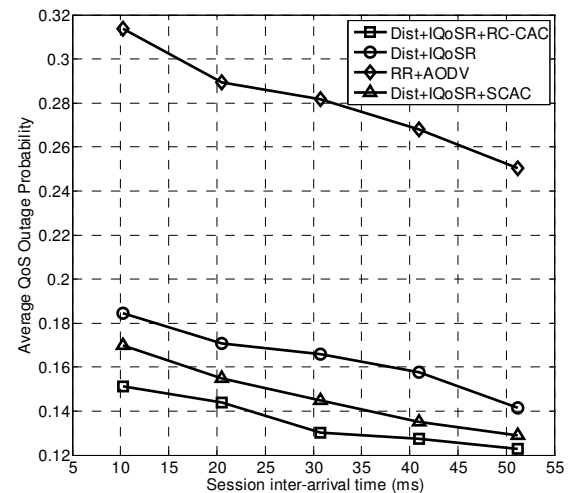

(b)

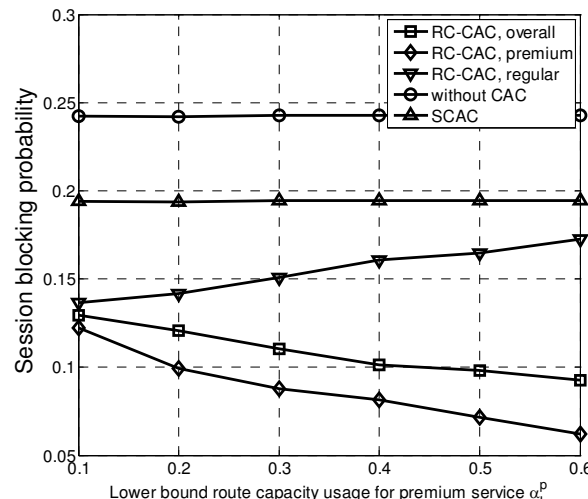

(C)

Fig. 3. Simulation results on (a) Gateway goodput (b) QoS outage probability per session value, for premium, regular and all completed sessions, both figures are plotted with respect to (w.r.t.) the new session inter-arrival time (c) Session blocking probability w.r.t. the lower route capacity usage bound $\alpha_{L}^{p}$ reserved for premium service users.

as the probability of any of the QoS requirements of a session to fail during the lifetime of the given session, or $\mathbf{I}_{s g}^{k}>1$. It is interesting to observe that for high network loading scenario, proposed joint algorithm can guarantee $85 \%$ of all sessions satisfying their all QoS requirements of the underlying application, as compared to $81 \%$ if no admission control is used, $82 \%$ if "SCAC" is used, and $68 \%$ for "RR+AODV". This is because the impact of new admitted session on existing flows has been estimated and accurately reflected during the route capacity estimation phase. Meanwhile, because no resource reservation is performed for premium users in other schemes, the flows admitted may exceed the network capacity which in turn jeopardize ongoing sessions' QoS.

Fig. 3c shows session blocking probability w.r.t. the lower bound route capacity usage $\alpha_{L}^{p}$ reserved for premium service users, while fixing the traffic input rate. As more resources are reserved, lower session blocking probability is expected; on the other hand, because overall route capacity is limited and shared among all regular and premium users, session blocking probability for regular users increases slightly from $14 \%$ to $16 \%$. Overall, the proposed joint algorithm achieve $10 \%$ and $15 \%$ lower blocking probabilities than "Dist+IQoSR+SCAC" and "Dist+IQoSR".

\section{CONCLUSIONS}

In this paper, we propose a novel admission control algorithm for multi-constrained QoS routing in wireless mesh networks. By using a generic mathematical function to represent the core resource manager for given candidate route, we study the analytical model to estimate the time varying "route capacity" for any combination of traffics with different QoS constraints. Then, we propose an admission control framework under multi-level grade-of-service scenario to make admission decision of the new flow. By maximizing the resource utilization within the route while minimizing the side effects on other joint/disjoint routes, extensive simulations show that the proposed cross-layer framework can successfully coordinate PHY, MAC and network layers to efficiently accommodate higher number of sessions in the network with satisfactory
QoS. Furthermore, it can successfully maintain reasonable low outage for the existing flows, and can guarantee the required GoS.

\section{REFERENCES}

[1] I. F. Akyildiz and X. Wang, "A survey on wireless mesh networks," IEEE Communications Magazine, vol. 43(9), pp. S23-S30, Sept. 2005.

[2] J. Ratica and L. Dobos, "Mobile ad-hoc networks connection admission control protocols overview," in Proceedings of 17th International Conference Radioelektronika, 2007, pp. 1-4.

[3] L. Seungjoon, G. Narlikar, M. Pal, G. Wilfong, and L. Zhang, "Admission control for multihop wireless backhaul networks with qos support," in Proceedings of IEEE WCNC, vol. 1, 2006, pp. 92-97.

[4] G. Narlikar, G. Wilfong, and L. Zhang, "Designing multihop wireless backhaul networks with delay guarantees," in Proceedings of IEEE INFOCOM 2006, pp. 1-12.

[5] D. Ghosh, A. Gupta, and P. Mohapatra, "Admission control and interference-aware scheduling in multi-hop wimax networks," in Proceedings of IEEE MASS 2007, pp. 1-9.

[6] T.-C. Tsai and C.-Y. Wang, "Routing and admission control in ieee 802.16 distributed mesh networks," in Proceedings of IFIP International Conference on Wireless and Optical Communications Networks (WOCN '07), 2007, pp. 1-5.

[7] S.-L. Su, Y.-W. Su, and J.-Y. Jung, "A novel qos admission control for ad hoc networks," in Proceedings of IFIP International Conference on Wireless and Optical Communications Networks (WOCN '07), 2007, pp. 4193-4197.

[8] H. Zhu, V. O. K. Li, Z. Ma, and M. Zhao, "Statistical connection admission control framework based on achievable capacity estimation," in Proceedings of IEEE ICC 2006, vol. 2, June 2006, pp. 748-753.

[9] C. H. Liu, K. K. Leung, and A. Gkelias, "A novel cross-layer QoS routing algorithm for wireless mesh network," in Proceedings of IEEE ICOIN 2008, Busan, Korea, 2008, pp. 1-5.

[10] C. H. Liu, A. Gkelias, and K. K. Leung, "A cross-layer framework of QoS routing and distributed scheduling for mesh networks," in Proceedings of IEEE VTC 2008 Spring, Singapore.

[11] Y. Hou and K. K. Leung, "A novel distributed scheduling algorithm for mesh networks," in Proceedings of IEEE Globecom 2007, U.S.A.

[12] OPNET Inc., http://www.opnet.com/.

[13] B. Sklar, "Rayleigh fading channels in mobile digital communication systems .I. Characterization," IEEE Communications Magazine, vol. 35, no. 7, pp. 90-100, Jul 1997.

[14] X. Yuan and Z. Duan, "Frr: a proportional and worst-case fair round robin scheduler," in Proceedings of IEEE INFOCOM 2005, vol. 2, U.S.A., pp. 831-842.

[15] C. Perkins and E. Royer, "Ad-hoc on-demand distance vector routing," in Proceedings of WMCSA'99, San Jose, CA, USA, 1999, pp. 90-100. 